Pacific Journal of Mathematics

EXPOSED POINTS OF CONVEX SETS

X 


\section{EXPOSED POINTS OF CONVEX SETS}

\section{Gustave Choquet, Harry Corson AND Victor KleE}

The two sections of this note are unrelated, except that both are concerned with the exposed points of a compact convex subset $K$ of a locally convex space $E$. In $\S 1$ it is proved that if $K$ is of finite dimension $d$, then the set of all its exposed points can be expressed as the union of a $G_{\delta}$ set, an $F_{\sigma}$ set, and $d-2$ sets each of which is the intersection of a $G_{\delta}$ set with an $F_{\sigma}$ set. A sharper assertion is proved for the threedimensional case, and some related results are obtained for certain infinite-dimensional situations. Section 2 describes a compact convex set in the space $\Re^{c}$ which has no algebraically exposed points. Both sections contain unsolved problems.

When $E$ is finite-dimensional, a point $p$ of $K$ is said to be exposed provided $\{\mathbf{p}\}$ is the intersection of $K$ with some supporting hyperplane of $K$, or, equivalently, provided there is a linear form on $E$ whose $K$-maximum is attained precisely at $p$. The set of all exposed points of $K$ will be denoted by exp $K$. It is well known that exp $K$ is a dense subset of ext $K$, the set of all extreme points of $K$. The set ext $K$ is a $G_{\delta}$ set, and in the two-dimensional case $\exp K$ is also a $G_{\delta}$ set. However, there are three-dimensional sets $K$ for which $\exp K$ is not a $G_{i}$ set $[1,4]$, and in Corson's example [1] the set exp $K$ is not even the union of a $G_{\delta}$ set and an $F_{\sigma}$ set. This suggests the problem of determining the Borel type of exp $K$, and the answer provided in $\S 1$ seems to be fairly complete.

For infinite-dimensional convex sets, the notion of exposed point may be defined in several different ways, all of which are equivalent in the finite-dimensional case. The weakest notion is that of an algebraically exposed point, this being a point of $K$ such that $p \in \exp (K \cap P)$ for every two-dimensional flat $P$ through $p$. The example in $\S 2$ provides a negative answer for questions raised by Phelps [7] and Klee [4].

1. The Borel type of the set of exposed points. Let us begin with the main finite-dimensional result.

THEOREM 1.1. Suppose that $K$ is a closed convex set of finite dimension d, and let $\exp K$ denote the set of all exposed points of $K$. Then

(a) if $d=2, \exp K$ is a $G_{\delta}$ set;

Received November 5, 1964. This paper was written at the University of Washington while the first author was a visiting Walker-Ames Professor and the others were supported by a grant from the National Science Foundation (NSF-GP-378). 
(b) if $d=3$, exp $K$ is the union of a $G_{\delta}$ sti and a set which is the intersection of $a G_{\delta}$ set and an $F_{\sigma}$ set;

(c) for arbitrary $d$, exp $K$ is the union of $a G_{\delta}$ set, an $F_{\sigma}$ set, and $d-2$ sets each of which is the intersection of a $G_{\delta}$ set with an $F_{\sigma}$ set.

Proof. For (a), it suffices to note that if $d=2$, then the set ext $K \sim \exp K$ is countable. For (b), let us define a face of a convex set $A$ as a maximal convex subset of the relative boundary of $A$. A face of dimension $j$ will be called a $j$-face. Let $S$ denote the union of all 0 -faces of $K$. Let $T$ denote the set of all points $x$ of $K$ such that $\{x\}$ is the intersection of a 1 -face or a 2 -face of $K$ with a supporting hyperplane of $K$. Finally, let $U$ denote the set of all endpoints of 1-faces of 2-faces of $K$. Then $S$ is a $G_{\delta}$ set, $T$ is an $F_{\sigma}$ set, and $U$ is countable. Further, $\exp K=S \cup(T \sim V)$ for some $V \subset U$, and the desired conclusion follows.

For an alternative proof, let $R$ denote the set of all points of $K$ at which $K$ admits a unique supporting hyperplane, let $S=R \cap \exp K$, let $T$ be the union of all 1 -faces of $K$ which are contained in $K \sim R$, and let $U$ be the set of all endpoints of these 1-faces. Then the above statements about $S, T$ and $U$ and still correct.

For (c), we assume that $K$ is a closed convex subset of Euclidean $d$-space $5^{d}$. Let $X$ denote the boundary of $K$ and let $Y$ denote the unit sphere $\left\{y \in \mathfrak{F}^{d}:\|y\|=1\right\}$. By means of the usual inner product, $Y$ will be regarded as a set of functions on $X$. For each point $x_{0}$ of $X$, we define

(1) $x_{0}^{s}=\left\{y_{0} \in Y: y_{0}\left(x_{0}\right)=\sup y_{0} X\right\}$

and for each point $y_{0}$ of $Y$ we define

(2) $y_{0}^{e}=\left\{x_{0} \in X: y_{0}\left(x_{0}\right)=\sup y_{0} X\right\}$.

A point $x$ of $K$ is exposed if and only if $x \in X$ and $\{x\}=y^{e}$ for some $y \in Y$.

In the sphere $Y$, we define an open j-ball of radius $\varepsilon$ as a set of the form $N(y, \varepsilon) \cap L \cap Y$, where $y \in Y, 0<\varepsilon<\sqrt{2}, N(y, \varepsilon)$ is the $\varepsilon$-neighborhood of $y$ in $\xi^{d}$, and $L$ is a $(j+1)$-dimensional linear subspace of $\mathfrak{F}^{d}$. Let $D(j, \varepsilon)$ denote the set of all points $x \in X$ such that $x^{s}$ contains an open $j$-ball of radius $\varepsilon$. Let $C_{1}, C_{2}, \cdots$ be a sequence of compact sets whose union is $X$, and let $Q(j, k, \varepsilon)$ denote the set of all points $x \in X$ for which $x^{s}$ contains an open $j$-ball $B$ of radius $\varepsilon$ such that for some $y \in B$, diam $y^{e} \geqq \varepsilon$ and $y^{e}$ intersects $C_{k}$. It can be verified that each of the sets $D(j, \varepsilon)$ and $Q(j, k, \varepsilon)$ is closed, and that each of the sets $Q(d-1, k, \varepsilon)$ is empty. The set $\exp K$ is the union of the $d$ sets

$$
\begin{aligned}
& X_{0}=D(0,1) \sim \bigcup_{k, \varepsilon} Q(0, k, \varepsilon), \\
& X_{j}=\bigcup_{\varepsilon} D(j, \varepsilon) \sim \bigcup_{k, \varepsilon} Q(j, k, \varepsilon) \quad(1 \leqq j \leqq d-2),
\end{aligned}
$$


and

$$
X_{d-1}=\bigcup_{\varepsilon} D(d-1, \varepsilon) \text {. }
$$

This completes the proof of 1.1 .

Problem 1.2. If $K$ is a three-dimensional compact convex set, must $\exp K$ be the intersection of $a G_{\delta}$ set and an $F_{\sigma}$ set $?^{1}$ If the answer is affirmative, what about the general finite-dimensional case?

In the remainder of this section, $X$ will denote a set and $Y$ will denote a set of real-valued functions on $X$. The sets $x_{0}^{s}\left(\right.$ for $x_{0} \in X$ ) and $y_{0}^{e}$ (for $y_{0} \in Y$ ) are defined as in (1) and (2) above. A point $x_{0}$ of $X$ will be called $Y$-smooth provided the set $x_{0}^{s}$ consists of a single point of $Y$; that is, provided precisely one member of $Y$ attains its maximum at $x_{0}$. And the point $x_{0}$ of $X$ will be called $Y$-exposed provided $\left\{x_{0}\right\}=$ $y_{0}^{e}$ for some $y_{0} \in Y$; that is, provided some member of $Y$ attains its maximum precisely at $x_{0}$. The sets of all $Y$-smooth and $Y$-exposed points of $X$ will be denoted respectively by $\operatorname{sm}_{Y} X$ and $\exp _{Y} X$.

The following remarks are elementary but useful. Their proofs are left to the reader.

LEMMA 1.3. If $Y$ is convex (with respect to the pointwise addition and scalar multiplication of real-valued functions on $X$ ), then the set $x^{s}$ is convex for each $x \in X$.

Lemma 1.4. Suppose that $x_{0}$ is a point of $X$, the functions $y_{1}, y_{2}, \cdots$ are members of $x_{0}^{s}$, the numbers $\lambda_{1}, \lambda_{2}, \cdots$ are strictly positive, and the series $\Sigma_{i} \lambda_{i} y_{i}$ is pointwise convergent to a function $y_{0} \in Y$. Then $y_{0} \in x_{0}^{s}$ and $y_{0}^{e}=\bigcap_{i} y_{i}^{e}$.

Lemma 1.5. Suppose that $X$ is a metric space, each member of $Y$ is upper semicontinuous on $X$, and $Y$ has the topology of uniform convergence on $X$. Then

(a) if $Y$ is compact, the set-valued transformation $x^{s} \mid x \in X$ is upper semicontinuous;

(b) if $X$ is compact, the set-valued transformation $y^{e} \mid y \in Y$ is upper semicontinuous.

When $X$ and $Y$ are as in 1.1, it follows from 1.1 that the set $\exp _{Y} X$ is both a $G_{\delta \sigma}$ set and an $F_{\sigma \delta}$ set. This may be extended in one direction as follows.

${ }^{1}$ If $K$ has no 2-faces, $\exp K$ is the union of a $G_{\delta}$ set and an $F_{\sigma}$ set, but the example of [1] shows that this is not true in general. 
Theorem 1.6. Suppose that $X$ is a compact metric space and $Y$ is a convex set of upper semicontinuous real-valued functions on $X$. Suppose that $Y$ is compact in the topology of uniform convergence. Then the set of all $Y$-exposed points of $X$ is an $F_{\sigma \delta}$ set.

Proof. Define

$$
M=\bigcup_{x \in X}\{x\} \times x^{s} \subset X \times Y,
$$

and for each $\varepsilon>0$ define

$$
W_{\varepsilon}=\left\{y \in Y: \operatorname{diam} y^{e}<\varepsilon\right\} .
$$

It follows from 1.5 (a) that $M$ is closed and from 1.5 (b) that $W_{\varepsilon}$ is open. Hence the set $M \cap\left(X \times W_{\varepsilon}\right)$ is an $F_{\sigma}$ subset of the compact set $X \times Y$, and its projection $A_{\varepsilon}$ on $X$ must also be an $F_{\sigma}$. To complete the proof it suffices to show that

$$
\exp _{Y} X=\bigcap_{\varepsilon>0} A_{\varepsilon}
$$

Inclusion in one direction is obvious. For the other, we consider an arbitrary point $x_{0} \in \bigcap_{\varepsilon>0} A_{\varepsilon}$ and want to show that $x_{0} \in \exp _{Y} X$. From the definition of $A_{\varepsilon}$ it follows that for each $\varepsilon>0$ there exists $y(\varepsilon) \in x_{0}^{s}$. such that diam $y(\varepsilon)^{e}<\varepsilon$. Since the set $x_{0}^{s}$ is convex by 1.3 and compact by 1.5 (a), it must include the function $y_{0}=\Sigma_{i} 2^{-i} y\left(2^{-i}\right)$. Then 1.4 implies that diam $y_{0}^{e}=0$, and the desired conclusion follows.

The remaining theorems of the present section are proved by refinements of the reasoning of 1.1 and 1.6. Indeed, 1.1 (c) could be derived as a corollary of 1.7 below $^{2}$, and 1.6 as a corollary of 1.9 . However, the simpler arguments were given first as an aid to clarity.

When $j$ is a nonnegative integer and $Z$ is a subset of a linear space $E$, the $j$-interior of $Z$ (int $Z$ ) is defined as the set of all points $z \in Z$ such that for some $j$-dimensional flat $F$ through $z, z$ is interior to the set $Z \cap F$ with respect to the natural topology of $F$.

Theorem 1.7. Suppose that $X$ and $Y$ are as in 1.6. For $j=$ $0,1, \cdots$, let $X_{j}$ denote the set of all points $x \in X$ such that $\operatorname{dim} x^{s} \geqq$ $j$ and $y^{e}=\{x\}$ for all $y \in$ int $^{j} x^{s}$. Then $X_{0}$ is a $G_{\delta}$ set and each of the sets $X_{1}, X_{2}, \cdots$ is the intersection of $a G_{\delta}$ set and an $F_{\sigma}$ set.

Proof. For the sake of simplicity, we assume at first that every

2 Of course, the Euclidean sphere $Y$ of 1.1 is not convex. However, it can be replaced in the proof of 1.1 by the boundary of a cube, which is the union of a finite number of convex sets. Another technical complication in deriving 1.1 directly from 1.7 would result from the fact that the set $X$ in 1.1 need not be compact but only $\sigma$-compact. 
member of $Y$ is bounded below as well as (automatically) above. Then $Y$ is a compact convex subset of the Banach space $E$ of all bounded real-valued functions on $X$. For $\varepsilon>0$ and for $j=0,1,2, \cdots$, let $D(j, \varepsilon)$ denote the set of all points $x \in X$ such that $x^{s}$ contains an open $j$-ball of radius $\varepsilon .^{3}$ Let $Q(j, \varepsilon)$ denote the set of all points $x \in X$ such that $x^{s}$ contains an open $j$-ball $B$ of radius $\varepsilon$ with diam $y^{e} \geqq \varepsilon$ for some $y \in B$. It is evident that

$$
X_{j}=\bigcup_{\varepsilon>0} D(j, \varepsilon) \sim \bigcup_{\varepsilon>0} Q(j, \varepsilon) .
$$

Further, $D(j, \varepsilon)$ and $Q(j, \varepsilon)$ are both antitone functions of $\varepsilon$, and $D(0, \varepsilon)=D(0,1)$ for all $\varepsilon>0$. Thus, it suffices to show that each of the sets $D(j, \varepsilon)$ and $Q(j, \varepsilon)$ is closed. This follows from 1.5, but the case in which $j \geqq 1$ may require some explanation.

Consider a sequence $x_{\alpha}$ of points of $X$, converging to a point $x_{0} \in X$. Suppose that $\left\{x_{1}, x_{2}, \cdots\right\} \subset D(j, \varepsilon)$. Then for each $i$ there are a point $z_{i} \in E$ and a $j$-dimensional linear subspace $L_{i}$ of $E$ such that

$$
z_{i}+N(0, \varepsilon) \cap L_{i} \subset x_{\imath}^{s} .
$$

For each $i, L_{i}$ admits a basis $\left\{b_{i}^{1}, \cdots, b_{i}^{j}\right\}$ consisting of points of norm $\varepsilon$ such that for $1<h \leqq j$, the point $b_{\imath}^{h}$ is at distance $\varepsilon$ from the linear hull of the set $\left\{b_{i}^{1}, \cdots, b_{i}^{h-1}\right\}_{0}^{4}$ The set $x_{0}^{s}$ is compact and (by 1.5 (a)) every neighborhood of $x_{0}^{s}$ contains all but finitely many of the sets $x_{i}^{s}$. Thus, we may assume without loss of generality that each of the sequences $z_{\alpha}$ and $b_{\alpha}^{h}$ is convergent; say $z_{\alpha} \rightarrow z_{0}$ and $b_{\alpha}^{h} \rightarrow b_{0}^{h}$. We claim that the set $\left\{b_{0}^{1}, \cdots, b_{0}^{j}\right\}$ is linearly independent. Indeed, suppose the contrary, whence for some $h>1$ we have $b_{0}^{h}=\Sigma_{1 \leqq r<h} \lambda_{r} b_{0}^{r}$. But then for each $i$,

$$
\begin{aligned}
\left\|b_{i}^{h}-\Sigma_{1 \leqq r<h} \lambda_{r} b_{i}^{r}\right\| & \leqq\left\|b_{i}^{h}-b_{0}^{h}\right\|+\left\|\Sigma_{1 \leqq r<h} \lambda_{r}\left(b_{0}^{r}-b_{i}^{r}\right)\right\| \\
& \leqq\left(1+\Sigma_{1 \leqq r<h}\left|\lambda_{r}\right|\right) \max _{1 \leqq r \leqq h}\left\|b_{i}^{h}-b_{0}^{h}\right\|,
\end{aligned}
$$

and for all sufficiently large $i$ this is inconsistent with the way in which the basis $\left\{b_{i}^{1}, \cdots, b_{i}^{j}\right\}$ was chosen. Thus the set $\left\{b_{0}^{1}, \cdots, b_{0}^{j}\right\}$ is linearly independent and its linear hull $L_{0}$ is a $j$-dimensional linear subspace of $E$. It is easily verified that

$$
z_{0}+N(0, \varepsilon) \cap L_{0} \subset x_{0}^{s},
$$

whence $x_{0} \in D(j, \varepsilon)$ and it follows that the set $D(j, \varepsilon)$ is closed.

Now suppose in addition that $\left\{x_{1}, x_{2}, \cdots\right\} \subset Q(j, \varepsilon)$. Then the points $z_{i}$ and linear subspaces $L_{i}$ can be chosen so that diam $y^{e} \geqq \varepsilon$ for some

${ }^{3}$ Here a $j$-ball of radius $\varepsilon$ is a set of the form $N(y, \varepsilon) \cap F$, where $F$ is a $j$-dimensional flat through $y$.

${ }^{4}$ This is an easy application of the Hahn-Banach theorem. 
$y \in z_{i}+N(0, \varepsilon) \cap L_{i}$. But then diam $z_{i}^{e} \geqq \varepsilon$, for it follows from 1.4 that $y^{e}$ is constant on every open segment contained in a set $x_{i}^{s}$ and hence on every open $j$-ball contained in $x_{\imath}^{s}$. With diam $z_{\imath}^{e} \geqq \varepsilon$ and $z_{\alpha} \rightarrow z_{0}$, it follows from 1.5 (b) that $\operatorname{diam} z_{0}^{e} \geqq \varepsilon$ and consequently the set $Q(j, \varepsilon)$ is closed.

Now to complete the proof of 1.7 , we abandon the assumption that all of the members of $Y$ are bounded. Let $S$ denote the linear space of all real-valued functions on $X$, and for $s_{1}, s_{2} \in S$ let

$$
\rho\left(s_{1}, s_{2}\right)=\sup _{x \in X}\left|s_{1}(x)-s_{2}(x)\right| \text {. }
$$

The function $\rho$ satisfies all of the requirements for a metric except that it may have the value $+\infty$. The $\rho$-topology for $S$ is the topology of uniform convergence. Let

$$
R=\left\{\left(s_{1}, s_{2}\right): \rho\left(s_{1}, s_{2}\right)<\infty\right\} \subset S \times S
$$

Then $R$ is an equivalence relation on $S$, and each equivalence class is both open and closed. Note that if $\left(s_{1}, s_{2}\right) \notin R$, then no two points of the segment $\left[s_{1}, s_{2}\right]$ are in the same equivalence class. Choose $y_{0} \in Y$ and let $\zeta(s)=s-y_{0}$ for all $s \in S$. Then $\zeta$ is an affine isometry of $S$ onto $S$, and $\zeta$ carries $Y$ into the Banach space $E$ of all bounded realvalued functions on $X$. From this point on, the proof is merely a paraphrase of the one already given when $Y \subset E$.

1.8. Corollary. Suppose that $X$ and $Y$ are as in 1.6. Then the set $\exp _{Y} X \cap \operatorname{sm}_{Y} X$ is a $G_{\delta}$ set, and the set $\left\{x \in \exp _{Y} X: \operatorname{dim} x^{s}<\infty\right\}$ is a $G_{\delta \sigma}$ set.

Proof. Note that the first set is equal to $X_{0} \sim \bigcup_{\varepsilon<0} D(1, \varepsilon)$ (in the notation of 1.7), while the second is equal to

$$
\bigcup_{j=0}^{\infty}\left(X_{j} \sim \bigcup_{\varepsilon>0} D(j+1, \varepsilon)\right) \text {. }
$$

For a shorter proof that $\exp _{Y} X \cap \operatorname{sm}_{Y} X$ is a $G_{\delta}$ set, let $M$ be as in 1.6 and let $N=\left\{(x, y) \in M\right.$ : $\left.\operatorname{diam} x^{s}=0=\operatorname{diam} y^{e}\right\}$. Then $N$ is a $G_{\delta}$ set and the projection of $N$ onto $X$ is a homeomorphism.

Our final aim in this section is to extend Theorem 1.6 and to apply the result thus obtained. For these purposes, we require some additional terminology. Suppose that $X$ is a set, $\boldsymbol{J}_{\alpha}$ is a sequence of classes of subsets of $X$, and $Y$ is a set of real-valued functions on $X$. A point $x_{0}$ of $X$ will be called $\left(Y, J_{\alpha}\right)$-exposed provided there exists $y_{0} \in x_{0}^{s}$ such that for each $i$ it is true that $\sup y_{0} J_{i}<y_{0}\left(x_{0}\right)$ for some $J_{i} \in J_{i}$. The 
set of all such points will be denoted by $\exp _{\left(Y, J_{\alpha}\right)} X$. If $X$ is a metric space, $\varepsilon_{\alpha}$ is a sequence of positive numbers converging to zero, and $\boldsymbol{J}_{i}$ is the set of all complements of open $\varepsilon_{i}$-neighborhoods of points of $X$, then a point $x_{0}$ is $\left(Y, \boldsymbol{J}_{\alpha}\right)$-exposed if and only if there exists $y_{0} \in Y$ such that the sets $\left\{x \in X: y_{0}(x)>y_{0}\left(x_{0}\right)-\varepsilon\right\}(\varepsilon>0)$ form a base of neighborhoods of $x_{0}$ in $X$. Such a point $x_{0}$ will be called strongly $Y$-exposed and the set of all such points will be denoted by $\operatorname{sexp}_{Y} X$. It is evident that $\operatorname{sexp}_{Y} X \subset \exp _{Y} X$, with equality when $X$ is compact and the members of $Y$ are all upper semicontinuous. Lindenstrauss [5] has an example in which $\operatorname{sexp}_{Y} X \neq \exp _{Y} X$ even though $X$ is a weakly compact convex subset of a Banach space $E$ and $Y$ is the conjugate space of $E$.

THEOREM 1.9. Suppose that $X$ is a metric space, $J_{\alpha}$ is a sequence of classes of subsets of $X, F$ is a complete metric linear space $e^{5}$ of real-valued functions on $X$, and $Y$ is a closed convex subset of $F$. Suppose that every member of $Y$ is upper semicontinuous on $X$, and that convergence in $Y$ implies uniform convergence on every member of $\bigcup_{i=1}^{\infty} \boldsymbol{J}_{i}$ as well as on every compact subset of $X$. Then

(a) if $Y$ is $\sigma$-compact, the set of all $\left(Y, J_{\alpha}\right)$-exposed points is an $F_{\sigma \delta}$ set in $X$;

(b) if $X$ is an analytic set and $Y$ is separable, the set of all its $\left(Y, J_{\alpha}\right)$-exposed is an analytic set. ${ }^{6}$

Proof. For each $i$, let $A_{i}$ denote the union of all sets $y^{e}$ such that for some set $J \in J_{i}$, sup $y J_{i}<\sup y X$. We claim that $\exp _{\left(Y, J_{\alpha}\right)} X=$ $\bigcap_{i=1}^{\infty} A_{i}$, where inclusion is obvious in one direction. For the reverse direction, let us consider an arbitrary point $x_{0} \in \bigcap_{i=1}^{\infty} A_{i}$. For each $i$, there exist $y_{i} \in Y$ and $J_{i} \in Y$ and $J_{i} \in J_{i}$ such that

$$
\sup y_{i} J_{i}<\sup y_{i} X=y_{i}\left(x_{0}\right) \text {. }
$$

We assume without loss of generality that the space $E$ is topologized by means of a metric $\rho$ which is not only complete but also translationinvariant [3]. Let the sequence of numbers $\lambda_{1}, \lambda_{2}, \cdots$ be such that always $0<\lambda_{i}<2^{-i}>\rho\left(0, \lambda_{i} y_{i}\right)$, whence the two series $\Sigma \lambda_{n}$ and $\Sigma \lambda_{n} y_{n}$ converge respectively to a number $\lambda \in[0,1]$ and a function $z \in F$. With $y_{0}=\lambda^{-1} z$, we have $y_{0} \in Y$, and since $\rho$-convergence implies pointwise convergence on $X$ it is evident that for each $i$,

$$
\sup y_{0} J_{i}<\sup y_{0} X=y_{0}\left(x_{0}\right) \text {. }
$$

Hence $x_{0} \in \exp _{\left(Y, J_{\alpha}\right)} X$.

Now let

${ }^{5}$ Addition and scalar multiplication in $F$ are assumed to be jointly continuous.

${ }^{6}$ An analytic set is a continuous image of a Borelian subset of the Hilbert cube. 


$$
M=\bigcup_{x \in X}\{x\} \times x^{s} \subset X \times Y,
$$

and for each $i$ let $W_{i}$ denote the set of all points $y \in Y$ such that

$$
\sup y J_{i}<\sup y X
$$

for some $J_{i} \in \boldsymbol{J}_{i}$. Since convergence in $Y$ implies pointwise convergence on $X$ as well as uniform convergence on every member of $\bigcup_{i=1}^{\infty} J_{i}$, each set $W_{i}$ is open. The set $M$ is closed, for if $\left(x_{1}, y_{1}\right),\left(x_{2}, y_{2}\right), \cdots$ is a sequence in $M$ with $x_{\alpha} \rightarrow x_{0}$ and $y_{\alpha} \rightarrow y_{0}$, then

$$
\begin{array}{r}
y_{0}\left(x_{0}\right) \underset{(1)}{\gtrless} \limsup _{i \rightarrow \infty} y_{0}\left(x_{i}\right) \underset{(2)}{=} \limsup _{i \rightarrow \infty} y_{i}\left(x_{i}\right) \\
\underset{(3)}{=} \limsup _{i \rightarrow \infty}\left(\sup y_{i} X\right) \underset{(4)}{\geqq} \sup y_{0} X .
\end{array}
$$

Here (1) is a consequence of the upper semicontinuity of $y_{0},(2)$ of the uniform convergence of $y_{\alpha}$ to $y_{0}$ on the compact set $\left\{x_{0}, x_{1}, x_{2}, \cdots\right\}$, (3) of the fact that $\left(x_{i}, y_{i}\right) \in M$, and (4) of the pointwise convergence of $y_{\alpha}$ to $y_{0}$ on $X$.

Since $M$ is closed and $W_{i}$ is open, the set $M \cap\left(X \times W_{i}\right)$ is an $F_{\sigma}$ subset of $X \times Y$. The projection of $M \cap\left(X \times W_{i}\right)$ on $X$ is exactly $A_{i}$. If $X$ is analytic and $Y$ is separable, then $X \times Y$ is analytic, whence each $A_{i}$ is an analytic set and thus the same is true of the set $\exp _{\left(Y, J_{\alpha}\right)} X$. If $Y$ is $\sigma$-compact then each set $A_{i}$ is an $F_{\sigma}$ set (and hence $\exp _{\left(Y, J_{\alpha}\right)} X$ is an $F_{\sigma \delta}$ set in $X$ ), for when $Z$ is a compact subset of $Y$ the projection on $X$ of a closed subset of $X \times Z$ must be closed in $X$. This completes the proof of 1.9 .

Corollary 1.10. Suppose that $X$ is a metric space, $C_{B}(X)$ is the Banach space of all bounded continuous real-valued functions on $X$ (in the topology of uniform convergence), and $Y$ is a closed convex subset of $C_{B}(X)$. Then the set $\operatorname{sexp}_{Y} X$ of all strongly $Y$-exposed points of $X$ is

an analytic set if $X$ is analytic and $Y$ is separable;

an $F_{\sigma \delta}$ subset of $X$ if $Y$ is $\sigma$-compact.

Proof. Apply 1.9, taking as $\boldsymbol{J}_{i}$ the set of all complements of open $2^{-i}$-neighborhoods of points of $X$.

Corollary 1.11. Suppose that $X$ is a locally compact separable metric space, $C(X)$ is the space of all continuous real-valued functions on $X$ (in the topology of uniform convergence on compact sets), and $Y$ is a closed convex subset of $C(X)$. Then the set $\exp _{Y} X$ of all $Y$-exposed points of $X$ is 
an analytic set if $Y$ is separable;

an $F_{\sigma \delta}$ subset of $X$ if $Y$ is $\sigma$-compact.

Proof. Let $X$ be metrized by means of a metric $\eta$ such that all $\eta$-bounded sets have compact closure. Then apply 1.9, taking as $\boldsymbol{J}_{i}$ the family of all sets of the form $\left\{x: 2^{-i} \leqq \eta\left(x, x_{0}\right) \leqq 2^{i}\right\}$ for $x_{0} \in X$.

CoRollary 1.12. Suppose that $E$ is a Banach space whose conjugate space $E^{\prime}$ is separable. If $X$ is a bounded analytic set in $E$, then $\operatorname{sexp}_{E^{\prime}} X$ is an analytic set. If $X$ is weatily compact, then both $\operatorname{sexp}_{E^{\prime}} X$ and $\exp _{E^{\prime}} X$ are analytic sets under the weak topology.

Proof. Apply 1.9 much as it was applied in 1.10 and 1.11, noting that convergence in $E^{\prime}$ implies uniform convergence on $X$, and that if $X$ is weakly compact, then (with $E^{\prime}$ separable) $X$ is metrizable under the weak topology.

When $X$ is a subset of a topological linear space $E$, a point $p$ of $X$ will be called topologically exposed provided there is a linear form $E$ whose restriction to $X$ is continuous and attains its maximum precisely at $p$. The following is an immediate consequence of 1.10 or 1.11.

Corollary 1.13. If $X$ is a metrizable compact subset of a topological linear space, then the set of all topologically exposed points of $X$ is an analytic set.

Problem 1.14. If $K$ is a compact convex subset of a Banach space $E$, must the set $\exp _{E^{\prime}} K$ be analytic or even Borelian?

2. A compact convex set having no algebraically exposed point. For some results and examples concerning the existence of exposed points of infinite-dimensional compact convex sets, see [4] and its references, and especially [5]. Here we shall construct a compact convex set which has no algebraically exposed point, thus settling problems raised in [4] (p. 97) and [7].

Proposition 2.1. Suppose that $I$ is an uncountable set of indices. Let $U=[-1,1]^{I}, V=\left\{x \in \mathfrak{R}^{I}: \Sigma_{i \in I} x_{i}^{2} \leqq 1\right\}$, and $K=U+V$. Then $K$ is a symmetric compact convex subset of the locally convex space $\mathfrak{R}^{I}$, but no point of $K$ is algebraically exposed.

Proof. Let $p=u+v$, with $u \in U$ and $v \in V$, and suppose that $p$ is an algebraically exposed point of $K$. Then of course, $p$ is an extreme point of $K$, and it follows that the points $u$ and $v$ are extreme in $U$ and 
$V$ respectively. Thus $u \in\{-1,1\}^{I}$, and since the sets $U$ and $V$ are both invariant with respect to permutation and change of sign of coordinates, we may assume without loss of generality that all the coordinates of $u$ are equal to 1 . Since the index set $I$ is uncountable and $\Sigma_{i \in I} v_{i}^{2}=1$, there exists $j \in I$ such that $v_{j}=0$. Let the point $w$ of $\Re^{I}$ be such that $w_{j}=1$ but $w_{i}=0$ for all $i \neq j$, and let $Q$ denote the plane consisting of all linear combinations of the points $v$ and $w$. Then the intersection $K \cap(p+Q)$ contains the circular disk $u+(V \cap Q)$ as well as the points $p$ and $p-w$. Since the line determined by $p-w$ and $p$ is tangent to the disk, $p$ cannot be an exposed point of the intersection $K \cap(p+Q)$. This completes the proof of 2.1.

If $I$ has the cardinality of the continuum, the space $\Re^{I}$ is separable (has a countable dense set) [6], but the set $K$ of 2.1 is not separable. We do not know of a separable compact convex set which has no algebraically exposed points.

A point $p$ of a convex set $X$ will be called an angular point of $K$ provided there exists a two-dimensional flat $P$ through $p$ such that the intersection $K \cap P$ is two-dimensional and has more than one line of support through $p$. As can be seen directly or by using the fact that the space $m(I)$ does not admit a smooth norm consistent with its topology [2], the set $K$ of 2.1 has many angular points. We do not know of a compact convex set which has neither angular points nor algebraically exposed points. A possible approach toward constructing such a set is indicated by the following remark.

Proposition 2.2. Let $K$ be a convex $F_{\sigma}$ set in a topological linear space $E$. Suppose that $K$ has no angular point and that no onepointed subset of $K$ is a $G_{\delta}$ set in $K$. Then no point of $K$ is algebraically exposed.

Proof. Supposing the contrary, we may assume without loss of generality that $E$ is the linear hull of $K$ and 0 is an algebraically exposed point of $K$. Let $M=\mathbf{U}_{\mu>0} \mu K$, an $F_{\sigma}$ set in $E$, and let $\boldsymbol{P}$ be the family of all two-dimensional linear subspaces of $E$ whose intersection with $K$ is also two-dimensional. For each $P \in \boldsymbol{P}, 0$ is an exposed point but not an angular point of the intersection $K \cap P$, and it follows readily that the set $(M \cap P) \sim\{0\}$ is an open halfplane in $P$. This implies that the set $M \sim\{0\}\left(=\bigcup_{P \in P}(M \cap P) \sim\{0\}\right)$ is an algebraically open halfspace in $E$, whence $M \sim\{0\}$ is the union of countably many translates of the $F_{\sigma}$ set $M$. But $M \sim\{0\}$ is an $F_{\sigma}$ set in $E$, whence $\{0\}$ is a $G_{\delta}$ set in $K$ and the contradiction completes the proof. 


\section{REFERENCES}

1. H. Corson, A compact convex set in $E^{3}$ whose exposed points are of the first category, Proc. Amer. Math. 16 (1965), 1015-1021.

2. M. M. Day, Strict convexity and smoothness of normed spaces, Trans. Amer. Math. Soc. 78 (1955), 516-528.

3. V. L. Klee, Invariant metrics in groups (Solution of a problem of Banach), Proc. Amer. Math. Soc, 3 (1952), 484-487.

4. - Extremal structure of convex sets. II, Math. Zeitschr. 69 (1958), 90-104.

5. J. Lindenstrauss, On operators which attain their norm, Israel J. Math. 1 (1963), 139-148.

6. E. Marczewski, Separabilité et multiplication cartésienne des espaces topologiques, Fund. Math. 34 (1947), 127-143.

7. R. R. Phelps, Unsolved problem in Proceedings of Symposia in Pure Math. 7 (Convexity), Amer. Math. Soc. Providence, R. I., (1963), p. 500.

Institut Henri Poincaré, Paris

University OF WASHington, SEATtLE 



\section{PACIFIC JOURNAL OF MATHEMATICS}

\section{EDITORS}

\section{H. SAMELSON}

Stanford University

Stanford, California

R. M. Blumenthal

University of Washington

Seattle, Washington 98105

\author{
*J. DugundJI \\ University of Southern California \\ Los Angeles, California 90007 \\ RICHARD ARENS \\ University of California \\ Los Angeles, California 90024
}

\section{ASSOCIATE EDITORS}
E. F. BECKENBACH
B. H. NeUManN
F. WolF
K. YosIDA

\section{SUPPORTING INSTITUTIONS}

UNIVERSITY OF BRITISH COLUMBIA
CALIFORNIA INSTITUTE OF TECHNOLOGY
UNIVERSITY OF CALIFORNIA
MONTANA STATE UNIVERSITY
UNIVERSITY OF NEVADA
NEW MEXICO STATE UNIVERSITY
OREGON STATE UNIVERSITY
UNIVERSITY OF OREGON
OSAKA UNIVERSITY
UNIVERSITY OF SOUTHERN CALIFORNIA

UNIVERSITY OF BRITISH COLUMBIA

UNIVERSITY OF CALIFORNIA

MONTANA STATE UNIVERSITY

NEW MEXICO STATE UNIVERSITY

OREGON STATE UNIVERSITY

OSAKA UNIVERSITY

UNIVERSITY OF SOUTHERN CALIFORNIA

\author{
STANFORD UNIVERSITY \\ UNIVERSITY OF TOKYO \\ UNIVERSITY OF UTAH \\ WASHINGTON STATE UNIVERSITY \\ UNIVERSITY OF WASHINGTON \\ AMERICAN MATHEMATICAL SOCIETY \\ CHEVRON RESEARCH CORPORATION \\ TRW SYSTEMS \\ NAVAL ORDNANCE TEST STATION
}




\section{Pacific Journal of Mathematics \\ Vol. 17, No. $1 \quad$ January, 1966}

Carlos Jorge Do Rego Borges, On stratifiable spaces ................ 1

Felix Earl Browder, Topological methods for non-linear elliptic equations of

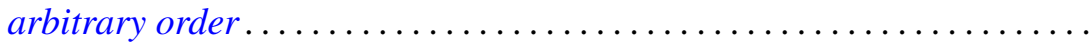

Gustave Choquet, Harry Corson and Victor Klee, Exposed points of convex

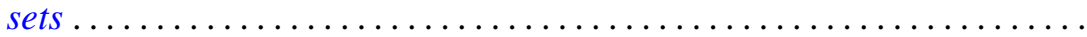

Phillip Emig, Remarks on the defect sum for a function meromorphic on an open Riemann surface ................................ 45

Ruth Goodman, A certain class of polynomials .................. 57

Sidney (Denny) L. Gulick, The bidual of a locally multiplicatively-convex

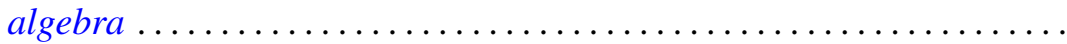

Eugene Carlyle Johnsen, Integral solutions to the incidence equation for finite projective plane cases of orders $n \equiv=2(\bmod 4) \ldots \ldots \ldots \ldots . .67$

Charles N. Kellogg, Centralizers and $H^{*}$-algebras .................. 121

Michael Lodato, On topologically induced generalized proximity relations. II .......................................... 131

P. H. Maserick, Half rings in linear spaces ..................... 137

Kathleen B O'Keefe, On a problem of J. F. Ritt .................... 149

Galen Lathrop Seever, Nonnegative projections on $C_{0}(X) \ldots \ldots \ldots \ldots$

Lawrence A. Shepp, Gaussian measures in function space ............ 167

Robert Charles Thompson, Classes of definite group matrices ........... 175 